\title{
PRODUÇÃO DE ENZIMAS PELO FUNGO Penicillium chrysogenum E UM FUNGO ISOLADO DA CASCA DO COCO (Aspergillus fumigatus) EM FSS UTILIZANDO RESÍDUO DE COCO COMO SUBSTRATO
}

\author{
S. D. de OLIVEIRA Jr ${ }^{1}$, P. F. de SOUZA FILHO ${ }^{1}$, G. R. MACEDO ${ }^{1}$, E. S. dos SANTOS $^{1}$, C. F. \\ ASSIS $^{2}$ \\ ${ }^{1}$ Universidade Federal do Rio Grande do Norte, Departamento de Engenharia Química \\ ${ }^{2}$ Universidade Federal do Rio Grande do Norte, Departamento de Farmácia \\ E-mail para contato: sergiodantas100@ hotmail.com
}

\begin{abstract}
RESUMO - O termo fermentação semi-sólida refere-se ao crescimento de microrganismos sobre um substrato sólido. O bagaço de coco (Cocos nucifera L.), um fruto encontrado principalmente na região Nordeste do Brasil, pode ser utilizado como substrato para este tipo de fermentação como um cultivo alternativo e de baixo custo econômico, sendo uma boa alternativa para o aproveitamento deste resíduo e com isso diminuindo danos ao meio ambiente. Este estudo avaliou o potencial deste resíduo como substrato para produção de enzimas celulolíticas em cultivo semi-sólido, utilizando os fungos Penicillium chrysogenum e Aspergillus fumigatus (isolado do resíduo da casca do coco). Avaliou-se as condições de cultivo (umidade e pH) utilizando um planejamento fatorial $2^{2}$ mais 3 pontos centrais. A máxima atividade enzimática para CMCase foi $0,282 \mathrm{UI} / \mathrm{mL}$ e para a Avicelase foi $0,018-0,020 \mathrm{UI} / \mathrm{mL}$ para as fermentações usando o fungo isolado. Já para o fungo Penicillium chrysogenum os valores encontrados para CMCase foi $0,233 \mathrm{UI} / \mathrm{mL}$ e para a Xilanase foi $0,735 \mathrm{UI} / \mathrm{mL}$. A fermentação foi realizada a $30{ }^{\circ} \mathrm{C}$ em BOD. Os dois fungos sintetizaram as enzimas sem a necessidade de qualquer indutor ou suprimento além da celulose presente no resíduo.
\end{abstract}

\section{INTRODUÇÃO}

O grande consumo de água de coco verde (in natura ou industrializada) vem aumentando a geração de resíduo desse fruto, o que acaba representando um grave problema quanto ao descarte desse material. $\mathrm{O}$ "coir" nome dado às fibras que constituem o mesocarpo grosso ou casca do coco (Cocos nucifera L.) é usado para produção de tapetes, esteiras e como substrato agrícola. As cascas de coco verde correspondem aproximadamente a $85 \%$ do peso bruto do fruto e a sua degradação leva em torno de 8 anos (Rosa et al., 2001). Este material é composto principalmente por celulose e hemicelulose.

Uma alternativa para utilizar esse material, que seria descartado e sem qualquer tipo de cuidado, é sua utilização como substrato para produção de enzimas por fermentação semi-sólida utilizando fungos filamentosos, por serem microrganismos que se adaptam a condições adversas, como baixa umidade, $\mathrm{pH}$ e temperaturas variadas. O processo de fermentação semi-sólida (FSS) envolve o crescimento e metabolismo de microrganismos na ausência ou quase ausência de água 
livre, empregando um substrato sólido, ou suporte. A FSS se apresenta como uma tecnologia para usar resíduos gerados como substratos, diminuindo possíveis problemas ambientais (Rocha, 2010). Esta técnica tem muitas vantagens sobre a fermentação submersa incluindo altos rendimentos e a baixa demanda de energia (Krishna, 2005).

O objetivo deste trabalho foi avaliar o efeito da umidade e do $\mathrm{pH}$ em fermentações para a produção de enzimas utilizando os fungos filamentosos Penicillium chrysogenum e um fungo isolado da casca do coco (Aspergillus fumigatus).

\section{MATERIAL E MÉTODOS}

\subsection{Bagaço de coco}

A casca e o mesocarpo fibroso foram utilizados para a obtenção do bagaço da casca de coco verde. Após a seleção dos cocos, as etapas do processo foram a dilaceração do material com facão, lavagem, secagem, moagem e armazenamento. A desidratação dos resíduos da casca de coco foi realizada em secador do tipo bandeja a $70{ }^{\circ} \mathrm{C}$ por cinco dias, triturado em moinho de facas tipo Willey e a fração foi coletada, sendo em seguida classificados em peneira de $20 \mathrm{mesh}$, e posteriormente, armazenados em sacos plásticos à temperatura ambiente.

\subsection{Caracterização química e físico-química do bagaço de coco verde}

Foi determinado o teor de extrativos (Pitarelo, 2007), polissacarídeos (celulose e hemicelulose) e lignina (Sluiter et al., 2011) e cinzas (Vasconcelos, 2012) da biomassa conforme os procedimentos descritos na literatura. A quantidade de pectina foi determinada pelo método gravimétrico por precipitação com pectato de cálcio descrito por Rangana (1979).

\subsection{Microrganismos utilizados nas fermentações}

Utilizou-se uma linhagem do fungo filamentoso Penicillium chrysogenum (807) da coleção ARS Culture Collection - BFPM Research Unit, National Center for Agriculture Utilization Research e um fungo isolado casca do coco.

\subsection{Isolamento de uma linhagem fúngica produtora de celulase}

Um fungo produtor de celulases foi isolado da casca de coco. A linhagem foi inoculada em frascos Erlenmeyer contendo caldo batata-dextrose e incubadas a $30{ }^{\circ} \mathrm{C}$ em Incubadora Refrigerada com Agitação orbital a 200 rpm (TE-421, TECNAL). Após 48 horas, as cepas foram transferidas para placas de Petri contendo ágar celulose com a seguinte composição: $0,5 \mathrm{~g} / \mathrm{L}$ de $\mathrm{MgSO}_{4}$ (sulfato de magnésio); 0,5 g/L de $\mathrm{KCl}$ (cloreto de potássio); 3,0 g/L de $\mathrm{NaNO} 3$ (nitrato de sódio); 0,01 g/L de $\mathrm{FeSO}_{4} .7 \mathrm{H}_{2} \mathrm{O}$ (sulfato de ferro); $1,0 \mathrm{~g} / \mathrm{L}$ de $\mathrm{K}_{2} \mathrm{HPO}_{4}$ (fosfato de potássio); 15 $\mathrm{g} / \mathrm{L}$ de ágar-ágar; $5,0 \mathrm{~g} / \mathrm{L}$ de celulose microcristalina. As placas foram incubadas a $30{ }^{\circ} \mathrm{C}$ por 120 horas seguindo metodologia descrita por Braga et al. (2009). O microrganismo foi identificado no laboratório BIOTRENDS, localizado em Fortaleza, sendo identificado como Aspergillus fumigatus. 


\subsection{Condições das fermentações}

As fermentações foram conduzidas em frascos Erlenmeyer de $250 \mathrm{~mL}$, cobertos com tampão de algodão. Foram pesados $5 \mathrm{~g}$ do substrato em base seca, adicionados $5 \mathrm{~mL}$ do inóculo com uma concentração de $1,0 \times 10^{6}$ esporos/mL (Coelho et al., 2001) e uma solução salina nutriente (sulfato de amônio $0,1 \%$, nitrato de amônio $0,1 \%$ e sulfato de magnésio heptahidratado $0,1 \%(\mathrm{p} / \mathrm{v})$, com $\mathrm{pH}$ corrigido para 3, 5 ou 7 (com ácido clorídrico $3 \mathrm{M}$ ou hidróxido de sódio $0,1 \mathrm{M}$ ) cujo volume variava de acordo com a umidade desejada (66, 70,5 e 75\%). Essas medições foram realizadas de acordo com o planejamento experimental. A mistura foi incubada em BOD a $30{ }^{\circ} \mathrm{C}$ e, após 120 horas de fermentação, foi realizada a etapa de extração do complexo enzimático.

O programa computacional STATISTICA@ (versão 7.0, StatSoft, Inc, 2004) foi utilizado para obtenção dos efeitos principais das variáveis e de suas interações, dos dados de análise de variância e para a obtenção de superfícies de resposta.

\subsection{Extração das enzimas}

A extração das enzimas ao término da fermentação foi realizada com adição de $4 \mathrm{~mL}$ de solução tampão acetato $200 \mathrm{mM}(\mathrm{pH} 4,5)$ por grama de bagaço utilizado no processo fermentativo, agitando-se manualmente com um bastão de vidro durante 1 hora em banho-maria à $30{ }^{\circ} \mathrm{C}$. O material foi filtrado com papel de filtro, em seguida foi centrifugado por 20 minutos a $3500 \mathrm{rpm}$ a $4{ }^{\circ} \mathrm{C}$. O sobrenadante foi recuperado e armazenado a $-18{ }^{\circ} \mathrm{C}$. Todos os ensaios foram realizados em duplicata.

\section{RESULTADOS E DISCUSSÃO}

A Tabela 1 representa a caracterização química e físico-química do bagaço do coco.

Tabela 1 - Caracterização química e físico-química do bagaço do coco

\begin{tabular}{cc}
\hline Análises & Bagaço do coco verde \\
\hline Teor de Umidade (\%) & $9,24 \pm 0,14$ \\
Teor de Extraíveis (\%) & $25,39 \pm 0,24$ \\
Teor de Cinzas (\%) & $0,46 \pm 0,02$ \\
Lignina Total (\%) & $36,23 \pm 0,12$ \\
Hemicelulose (\%) & $23,79 \pm 0,31$ \\
Celulose (\%) & $39,09 \pm 0,48$ \\
Pectina (\%) & $1,64 \pm 0,06$ \\
\hline
\end{tabular}

O teor de extraíveis do material vegetal, composto por ceras, álcoois, lipídeos, esteroides, ácidos graxos, hidrocarbonetos, flavonoides etc., foi de 25,39\%. O restante dos materiais é considerado substâncias não extrativas, pois compõem as cinzas que restam quando a matéria orgânica é queimada. O teor de cinza do bagaço do coco foi de $0,46 \%$. A composição do bagaço do coco de acordo com Rosa et al, (2001) apresentou as seguintes porcentagens de lignina (35 a $45 \%$ ), celulose (23 a 43\%) e de hemicelulose (3 a 12\%), comparando com os resultados obtidos com o do autor, confirma-se que o bagaço de coco é material lignocelulósico com potencial para ser utilizado como substrato, apresentando uma porcentagem de celulose de 39,09\%, de hemicelulose de $23,79 \%$ e o teor de lignina total encontrado foi $36,23 \%$. 


\subsection{Valores das ativadades enzimáticas em função da umidade e $\mathbf{p H}$, usando um fungo isolado (Aspergillus fumigatus) da casca do coco nas fermentações com o bagaço do coco como substrato.}

A Tabela 2 apresenta o planejamento experimental e os resultados obtidos de atividade celulolítica do extrato enzimático produzido pelo fungo isolado por fermentação semi sólida, em função da umidade e do $\mathrm{pH}$, dos quais foram avaliados as atividades enzimáticas CMCase e Avicelase.

Tabela 2 - Planejamento fatorial $\left(2^{2}\right)$ e resultados das atividades enzimáticas CMCase e Avicelase produzida pelo fungo isolado (Aspergillus fumigatus) em fermentação semi sólida.

\begin{tabular}{ccccc}
\hline Experimentos & $\mathrm{pH}$ & Umidade $(\%)$ & A. E. CMCase (UI/mL) & A. E. Avicelase (UI/mL) \\
\hline 1 & 3 & 66 & $0,282 \pm 0,002$ & $0,011 \pm 0,002$ \\
2 & 3 & 75 & $0,252 \pm 0,003$ & $0,013 \pm 0,021$ \\
3 & 7 & 66 & $0,279 \pm 0,006$ & $0,010 \pm 0,001$ \\
4 & 7 & 75 & $0,267 \pm 0,023$ & $0,010 \pm 0,021$ \\
$5^{*}$ & 5 & 70,5 & $0,216 \pm 0,001$ & $0,018 \pm 0,008$ \\
$6^{*}$ & 5 & 70,5 & $0,218 \pm 0,003$ & $0,019 \pm 0,003$ \\
$7^{*}$ & 5 & 70,5 & $0,224 \pm 0,002$ & $0,020 \pm 0,004$ \\
\hline
\end{tabular}

*Ponto central

De acordo com o diagrama de Pareto da Figura 1A, para atividade CMCase, apenas a umidade é estatisticamente significativa para um nível de $95 \%$ de confiança. $\mathrm{O}$ pH e a interação entre as duas variáveis não influenciaram na produção da CMCase. Pode-se, portanto, apontar a umidade como um parâmetro importante para a síntese da enzima. $\mathrm{O}$ mesmo não ocorre para o diagrama de Pareto da Figura 1B para atividade de Avicelase. Nenhum dos efeitos nem a interação entre eles foram significativos, mostrando assim que os fatores estudados não influenciam a produção desta enzima dentro das condições estudadas.
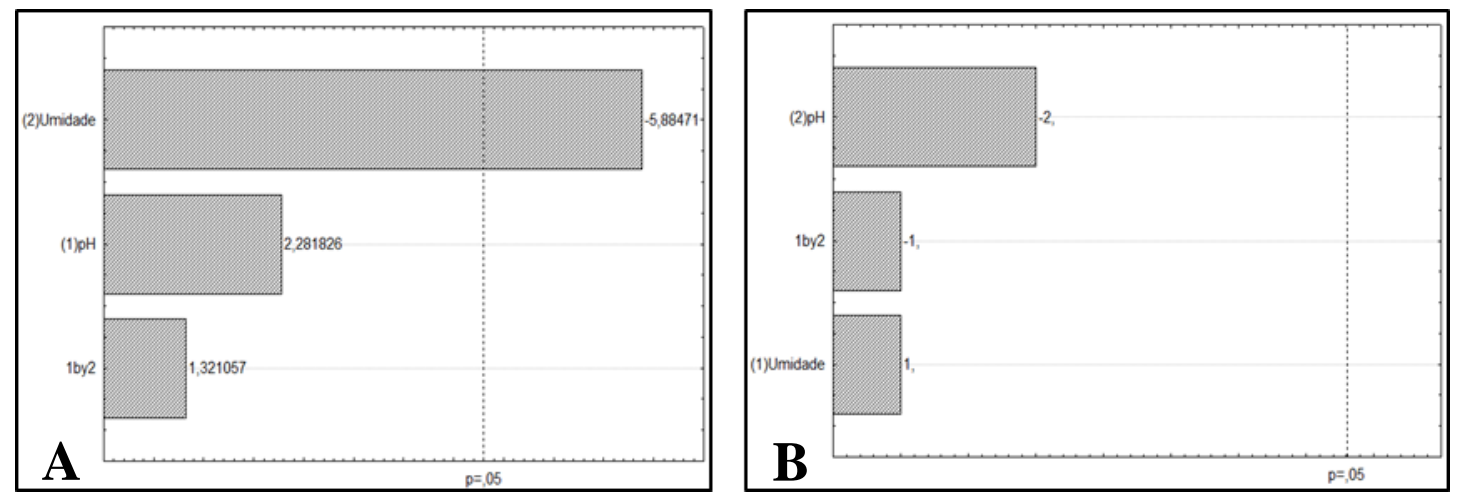

Figura 1 - Gráficos de Pareto do pH, umidade e da interação entre eles, para as atividades da CMCase (A) e Avicelase (B) para o fungo isolado (Aspergillus fumigatus) usando o bagaço do coco como substrato. 
As fermentações ocorreram com a temperatura fixa $\left(30{ }^{\circ} \mathrm{C}\right)$. As melhores condições apresentadas para a produção da enzima CMCase foram dos experimentos 1 e 3 . A umidade de $66 \%$ consistiu no melhor resultado para a produção da enzima CMCase, com atividades enzimáticas de 0,282 e 0,279 UI/mL. No caso da atividade da Avicelase os experimentos que apresentaram a melhor produção da enzima foram os 5, 6 e 7, ou seja, os pontos centrais, apresentando os valores de 0,018, 0,019 e 0,020 UI/mL, neste caso a umidade e pH intermediários (70,5\% e 5, respectivamente) resultaram em uma maior produção da enzima. Observa-se pouca inclinação da superfície de resposta (Figura 2), indicando que os efeitos não são significativos, para $95 \%$ de confiança, para os níveis avaliados.
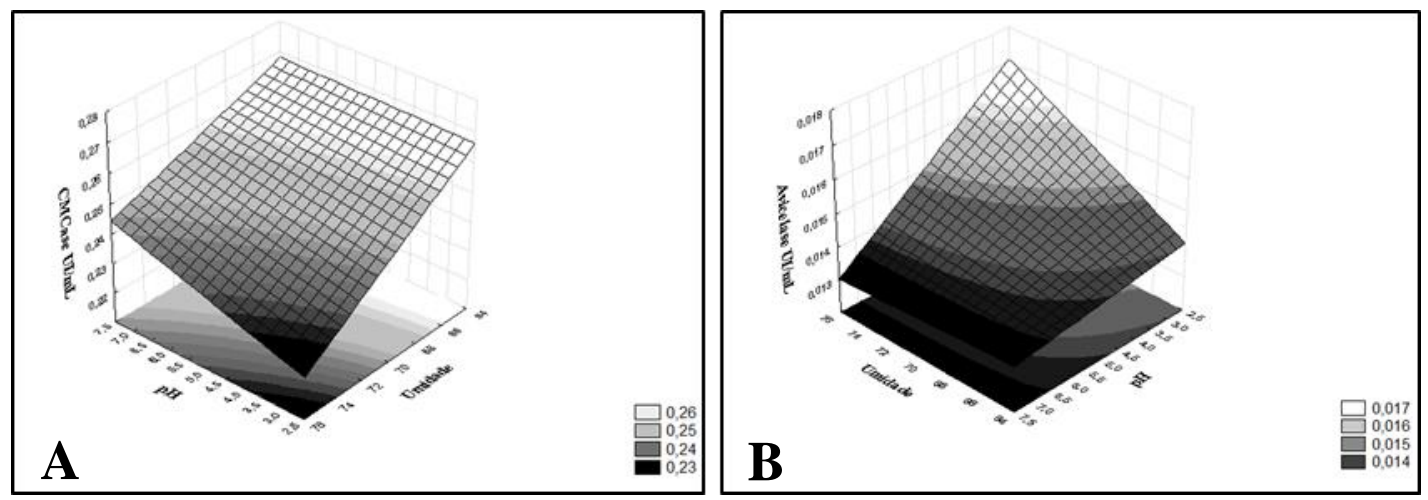

Figura 2 - Superfícies de resposta para a determinação das atividades enzimáticas CMCase (A) e Avicelase (B) para o fungo isolado (Aspergillus fumigatus) usando o bagaço do coco como substrato.

\subsection{Valores das ativadades enzimáticas em função da umidade e pH, usando o Penicillium chrysogenum nas fermentações com o bagaço do coco como substrato.}

A Tabela 3 apresenta o planejamento experimental e os resultados obtidos de atividade celulolítica do extrato enzimático produzido pelo fungo Penicillium chrysogenum por fermentação semi-sólida, em função da umidade e do $\mathrm{pH}$, dos quais foram realizados ensaios das atividade enzimáticas CMCase e Xilanase usando o bagaço do coco no tempo de 120 horas e na temperatura de $30{ }^{\circ} \mathrm{C}$.

Tabela 3 - Planejamento fatorial $\left(2^{2}\right)$ e resultados das atividades enzimáticas CMCase e Xilanase produzida pelo fungo Penicillim chrysogenum em fermentação semi sólida.

\begin{tabular}{ccccc}
\hline Experimentos & $\mathrm{pH}$ & Umidade(\%) & A.E. CMCase $(\mathrm{UI} / \mathrm{ml})$ & A.E. Xilanase $(\mathrm{UI} / \mathrm{ml})$ \\
\hline 1 & 3 & 66 & $0,067 \pm 0,0084$ & $0,541 \pm 0,0078$ \\
2 & 3 & 75 & $0,029 \pm 0,0084$ & $0,588 \pm 0,011$ \\
3 & 7 & 66 & $0,176 \pm 0,011$ & $0,513 \pm 0,0035$ \\
4 & 7 & 75 & $0,233 \pm 0,0084$ & $0,735 \pm 0,0014$ \\
$5^{*}$ & 5 & 70,5 & $0,049 \pm 0,0021$ & $0,611 \pm 0,0021$ \\
$6^{*}$ & 5 & 70,5 & $0,055 \pm 0,0007$ & $0,608 \pm 0,0056$ \\
$7^{*}$ & 5 & 70,5 & $0,052 \pm 0,0028$ & $0,619 \pm 0,0014$ \\
\hline
\end{tabular}

*Ponto central 
De acordo com o diagrama de Pareto da Figura 3A para atividade da CMCase, o pH e a interação entre os parâmetros é significativa para um nível de $95 \%$ de confiança. A umidade não influenciou de maneira significativa para a produção dessa enzima nessas condições. Já para atividade da Xilanase, como é representado na Figura 3B, o pH, a umidade e a interação entre estes influenciaram de maneira significativa para a produção desta enzima.
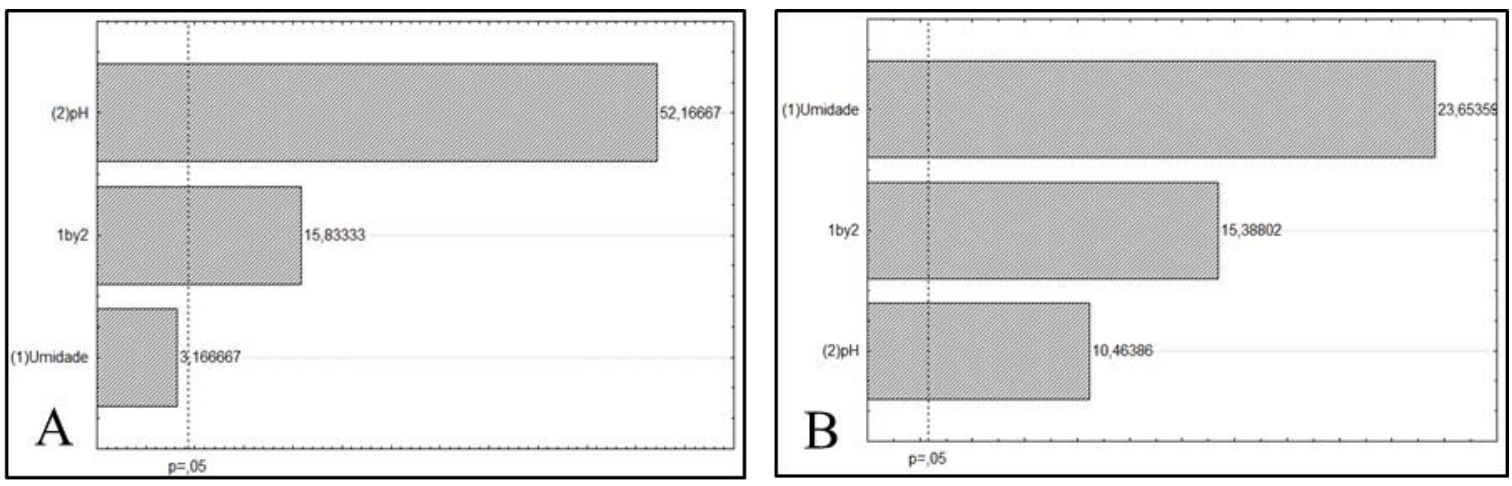

Figura 3 - Gráficos de Pareto do pH, umidade e da interação entre eles, para as atividades da CMCase (A) e Xilanase (B) para o fungo Penicillium chrysogenum usando o bagaço do coco como subtrato.

Os maiores valores atingidos para as atividades enzimáticas de CMCase e Xilanase foram obtidos em valores de umidade de $75 \%$ para o bagaço do coco como sustrato. Confirmando novamente que a umidade é um parâmetro importante para a produção das enzimas.

A Figura 4A ilustra o efeito das combinações de variáveis independentes $\mathrm{pH}$ e umidade sobre a atividade da enzima CMCase, a atividade enzimática máxima foi de $0,233 \mathrm{UI} / \mathrm{mL}$ o valor correspondente ao experimento 4, onde a umidade utilizada foi $75 \%$ e com $\mathrm{pH} 7$. Já para a produção da Xilanase (Figura 8), a atividade enzimática máxima foi de $0,735 \mathrm{UI} / \mathrm{mL}$ os valores correspondente ao experimento 4, onde a umidade utilizada foi $75 \%$ e com $\mathrm{pH} 7$.
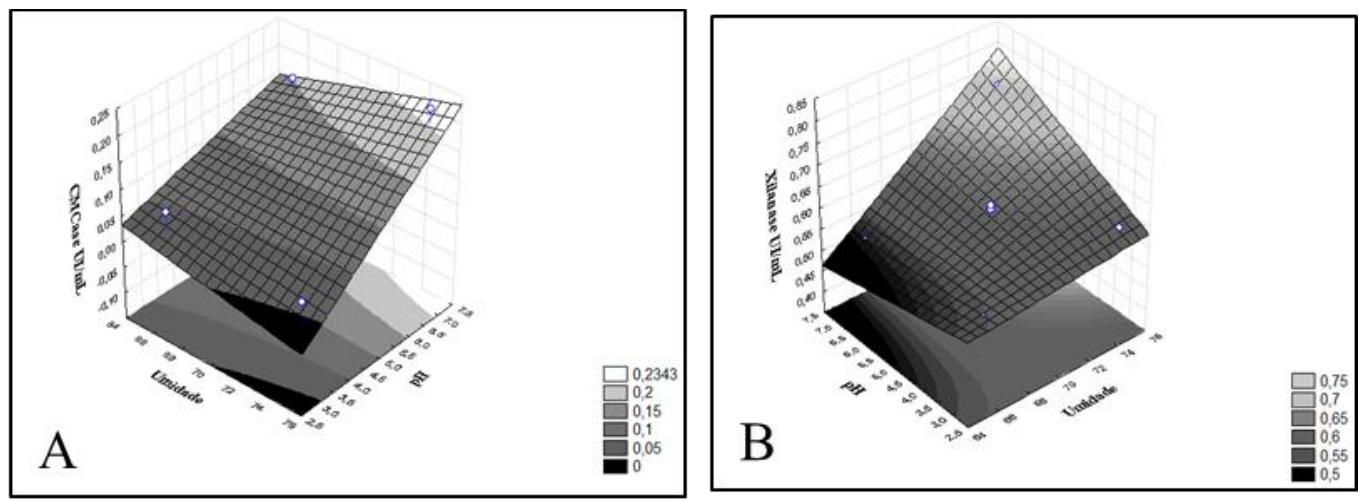

Figura 4 - Superfícies de resposta para a determinação das atividades enzimáticas CMCase (A) e Avicelase (B) para o fungo Penicillium chrysogenum usando o bagaço do coco como substrato.

\section{CONCLUSÕES}


A caracterização química e físico-química do resíduo bagaço do coco indicou seu potencial como substrato para produção de enzimas. O fungo isolado (Aspergillus fumigatus) apresentou potencial para a produção das enzimas CMCase e Avicelase, já o fungo Penicillium chrysogenum apresentou atividades enzimáticas CMCase e Xilanase utilizando o bagaço do coco como substrato no tempo de 120 horas e na temperatura de $30^{\circ} \mathrm{C}$.

\section{REFERÊNCIAS}

BRAGA, R. M., et al. Avaliação da produção de celulase por cepas de Fusarium. In: Simpósio Nacional de Bioprocessos, 17., 2009, Natal. Anais.Natal, 2009.

COELHO, M. A. Z. et al. Aproveitamento de resíduos agroindustriais: produção de enzimas a partir da casca de coco verde. Boletim Ceppa, Curitiba, v. 19, n. 1, p. 33-42, 2001.

PITARELO, A. P. Avaliação da susceptibilidade do bagaço e da palha de cana-de-açúcar à bioconversão via pré-tratamento a vapor e hidrólise enzimática. 2007, 142f. Dissertação (Mestrado em Química) - Universidade Federal do Paraná, Curitiba.

RANGANA, S. Manual of analysis of fruits and vegetable products. New Delhi: Tata McGrawHill, 1979. 634p.

ROCHA, C.P. Otimização da produção de enzimas por Aspergillus niger em Fermentação em estado sólido. 2010. 136f. Dissertação de Mestrado. Programa de Pós-Graduação emEngenharia Química. Universidade Federal de Uberlândia, Uberlândia, 2010.

ROSA, M. de F.; SEIXAS, F. J de S.; MONTENEGRO, A.A. T.; ABREU, F. A. P de.; CORREIA, D.; ARAÚJO, F. B.; NORÕES, E.R. de V. Caracterização do pó da casca de coco verde usado comosubstrato agrícola. Comunicado Técnico Embrapa Agroindústria Tropical, N 54, p.1-6,maio 2001.

SLUITER, A.; HAMES, B.; RUIZ, R.; SCARLATA, C.; SLUITER, J.; TEMPLETON, D.; CROCKER, D. Determination of structural carbohydrates and lignin in biomass. Golden, CO: National Renewable Energy Laboratory, 2011.

VASCONCELOS, S. M. Pré-tratamento de bagaço de cana-de-açúcar com ácido fosfórico diluído para aplicação em biorrefinarias. 2012. 184f. Tese (Doutorado em Engenharia Química) Programa de Pós-Graduação em Engenharia Química, Universidade Federal de Pernambuco, Recife. 